\title{
A Comparative Study of Algorithms for Computing Continued Fractions of Algebraic Numbers
}

\author{
Richard P. Brent ${ }^{1}$, Alfred J. van der Poorten ${ }^{2}$ and Herman J. J. te Riele ${ }^{3}$ \\ 1 Computer Sciences Laboratory, \\ Research School of Information Sciences and Engineering, \\ Australian National University, Canberra, ACT 0200 Australia \\ rpb@nimbus.anu.edu.au \\ 2 ceNTRe for Number Theory Research, \\ School of Mathematics, Physics, Computing and Electronics, \\ Macquarie University, Sydney NSW 2109 Australia \\ alf@mpce.mq.edu.au \\ 3 CWI, Department of Numerical Mathematics, \\ Kruislaan 413, 1098 SJ Amsterdam, The Netherlands \\ herman@cwi.nl
}

\section{Introduction}

The naïve way to compute the continued fraction of a real number $\alpha>1$ is to find a very accurate numerical approximation to $\alpha$, and then to iterate the well known truncate-and-invert step which computes the next partial quotient $a=\lfloor\alpha\rfloor$ and the next complete quotient $\alpha^{\prime}=1 /(\alpha-a)$. We call this the basic method. In the course of this process precision is lost, and one has to take precautions to stop before the partial quotients become incorrect. Lehmer [7] gives a safe stopping criterion, and a trick to reduce the amount of multilength arithmetic, leading to the so-called indirect method [14]. Schönhage [13] describes an algorithm for computing the greatest common divisor of $u$ and $v$, and the related continued fraction expansion of $u / v$, in $O\left(n \log ^{2} n \log \log n\right)$ steps if neither $u$ nor $v$ exceed $2^{n}$.

A disadvantage of the basic method is that if one wishes to extend the list of partial quotients computed from an initial approximation of $\alpha$, one has to compute a more accurate initial approximation of $\alpha$, compute the new complete quotient using this new approximation and the partial quotients already computed from the old approximation, and then extend the list of partial quotients using that new complete quotient (we notice that Shiu in $[14, \mathrm{p} .1312]$ slips in suggesting that all the previous calculations have to be repeated; of course the partial quotients already computed do not have to be recomputed).

Bombieri and Van der Poorten [2], and Shiu [14], have recently recalled a remedy for this problem. They give a formula for computing a rational approximation of the next complete convergent from the first $n$ partial quotients. From 
that complete convergent some $n$ new partial quotients can be computed. So each step provides an approximate doubling of the number of partial quotients. Shiu calls this the direct method. To start the direct method, a few partial quotients are computed with the basic method. In [2] this approach is proposed for algebraic numbers (zeros of polynomials defined over $\mathbb{Z}$ ) of degree $\geq 3$, whereas Shiu also applies it to more general numbers, namely to transcendental numbers defined as zeros of functions for which the logarithmic derivative at some rational point can be computed with arbitrary precision. This includes numbers such as $\pi, \log \pi$, and $\log 2$. For each of thirteen different such numbers Shiu computes 10000 partial quotients. Their frequency distributions are compared with the one which almost all numbers should obey according to the Khintchine-Lévy theory $[4,8]$. No significant deviations from this theory are reported.

Curiously, Shiu does not refer to what we will call the polynomial method for algebraic numbers $[3,6,12]$ of degree $\geq 3$, which computes the partial quotients of $\alpha$ using only the coefficients of its defining polynomial. Moreover, Shiu gives neither implementational details of his direct method, nor of the indirect method mentioned above (which he applies to four numbers which cannot be handled by the direct method). He concludes that his direct method is "superior in the sense that the computing times for a modest number of partial quotients using the indirect and the direct method are similar, whereas it becomes prohibitively long for the basic algorithm".

This is not quite a reproducible conclusion. Moreover, the polynomial method is not included in Shiu's study. This motivated us to produce a more explicit comparison of the various methods. We have used the occasion to compute some 200000 partial quotients of six different algebraic numbers and to test those collections of partial quotients against the expectation for the partial quotients of 'random' real numbers.

A second motivation for the present study is the use of the continued fraction expansion of algebraic numbers in solution methods for certain diophantine inequalities. For example, in [11] the Diophantine inequality

$$
\left|x^{3}+x^{2} y-2 x y^{2}-y^{3}\right| \leq 200
$$

known to have just finitely many integral solution pairs $(x, y)$, is solved for $|y| \leq 10^{500}$ with the help of the computation of a (modest) number of partial quotients of the continued fraction expansion of one of the real roots of the third degree polynomial $x^{3}+x^{2}-2 x-1 .^{1}$

\footnotetext{
${ }^{1}$ Recently, De Weger [16] has determined the complete set of rational integers $x, y$ that satisfy the inequality $\left|x^{3}+x^{2} y-2 x y^{2}-y^{3}\right| \leq 10^{6}$ (without an a priori bound on $|y|$ ).
} 


\section{Notation and Error Control}

\subsection{Notation}

Let $\alpha$ be a real number $>1$. The continued fraction expansion of $\alpha$ is defined by

$$
\alpha=a_{0}+\frac{1}{a_{1}+\frac{1}{a_{2}+\frac{1}{\ddots}}}
$$

where $a_{i}=\left\lfloor\alpha_{i}\right\rfloor, \alpha_{i+1}=1 /\left(\alpha_{i}-a_{i}\right), i=0,1, \ldots$, with $\alpha_{0}=\alpha$. The positive integers $a_{0}, a_{1}, \ldots$ are called the partial quotients of $\alpha$ and the real numbers $\alpha_{i}$ are referred to as its complete quotients. It is convenient to write

$$
\alpha=\left[a_{0}, a_{1}, a_{2}, \ldots\right]=\left[a_{0}, a_{1}, \ldots, a_{n}, \alpha_{n+1}\right],
$$

where $\alpha_{n+1}=\left[a_{n+1}, a_{n+2}, \ldots\right]$.

If $\alpha$ is rational, say $\alpha=u / v$, then its continued fraction expansion terminates (with some $\alpha_{i}=0$ ) and the basic method is nothing other than the Euclidean algorithm for computing the greatest common divisor of $u$ and $v$.

The rational approximation

$$
\left[a_{0}, a_{1}, \ldots, a_{n}\right]=\frac{p_{n}}{q_{n}}
$$

of $\alpha$ is called the $n$-th convergent of $\alpha$. The numerators and denominators of these approximations are computed by the formulas

$$
\left.\begin{array}{rl}
p_{i+1} & =a_{i+1} p_{i}+p_{i-1} \\
q_{i+1} & =a_{i+1} q_{i}+q_{i-1}
\end{array}\right\} \quad i=0,1, \ldots,
$$

where $p_{0}=a_{0}, q_{0}=1, p_{-1}=1$, and $q_{-1}=0$. In matrix notation, this is

$$
\left(\begin{array}{cc}
p_{i+1} & p_{i} \\
q_{i+1} & q_{i}
\end{array}\right)=\left(\begin{array}{cc}
a_{0} & 1 \\
1 & 0
\end{array}\right)\left(\begin{array}{cc}
a_{1} & 1 \\
1 & 0
\end{array}\right) \ldots\left(\begin{array}{cc}
a_{i+1} & 1 \\
1 & 0
\end{array}\right),
$$

which entails, on taking determinants, that

$$
p_{i+1} q_{i}-p_{i} q_{i+1}=(-1)^{i}, \quad i=0,1, \ldots
$$

\subsection{Error Control}

When one computes the partial quotients $a_{0}, a_{1}, \ldots$ from a numerical approximation $\bar{\alpha}$ of $\alpha$, one loses precision. The error can be controlled with the help of the following two lemmas. Lemma 1 gives a sufficient condition for $\lfloor\bar{\alpha}\rfloor=\lfloor\alpha\rfloor$ to be true, and Lemma 2 gives an upper bound for the relative error in $\bar{\alpha}^{\prime}=1 /(\bar{\alpha}-\lfloor\bar{\alpha}\rfloor)$ as a function of $\bar{\alpha}$, the relative error in $\bar{\alpha}$, and $\bar{\alpha}^{\prime}$. 
Lemma 1 Let $\bar{\alpha}>0$ be a numerical (rational) approximation of $\alpha>0$ with relative error bounded by $\delta$, i.e., $\bar{\alpha}=\alpha(1+\epsilon)$ with $|\epsilon|<\delta$. If

$$
(\lfloor\bar{\alpha}\rfloor+1) \delta<\bar{\alpha}-\lfloor\bar{\alpha}\rfloor<1-(\lfloor\bar{\alpha}\rfloor+1) \delta,
$$

then $\lfloor\bar{\alpha}\rfloor=\lfloor\alpha\rfloor$.

Proof. We show that $\lfloor\bar{\alpha}\rfloor<\alpha<\lfloor\bar{\alpha}\rfloor+1$. Since $\bar{\alpha}>0$, it follows from (2) that $\delta<1 / 2$.

Firșt, since $1-\delta<1 /(1+\epsilon)$ for $\delta<1$, we have

$$
\bar{\alpha}(1-\delta)<\frac{\bar{\alpha}}{1+\epsilon}=\alpha .
$$

Furthermore, $\bar{\alpha} \delta<(\lfloor\bar{\alpha}\rfloor+1) \delta$ so that, by the left inequality in (2), $\bar{\alpha} \delta<\bar{\alpha}-\lfloor\bar{\alpha}\rfloor$. With the above inequality this implies that $\lfloor\bar{\alpha}\rfloor<\bar{\alpha}(1-\delta)<\alpha$.

Second, since $1 /(1+\epsilon)<1 /(1-\delta)$ for $\delta<1$, we have

$$
\alpha=\frac{\bar{\alpha}}{1+\epsilon}<\frac{\bar{\alpha}}{1-\delta} .
$$

From the right inequality in $(2), \bar{\alpha}<(\lfloor\bar{\alpha}\rfloor+1)(1-\delta)$, so that $\bar{\alpha} /(1-\delta)<\lfloor\bar{\alpha}\rfloor+1$.

Lemma 2 Suppose the conditions of Lemma 1 hold, and let

$$
\alpha^{\prime}=\frac{1}{\alpha-\lfloor\alpha\rfloor}, \quad \bar{\alpha}^{\prime}=\frac{1}{\bar{\alpha}-\lfloor\bar{\alpha}\rfloor} .
$$

Then an upper bound for the relative error in $\bar{\alpha}^{\prime}$ with respect to $\alpha^{\prime}$ is given by $\bar{\alpha} \bar{\alpha}^{\prime} \delta /(1-\delta)$.

Proof. We have, for $\delta<1 / 2$,

$$
\begin{gathered}
\left|\frac{\bar{\alpha}^{\prime}-\alpha^{\prime}}{\alpha^{\prime}}\right|=\left|\frac{\frac{1}{\bar{\alpha}-\lfloor\bar{\alpha}\rfloor}-\frac{1}{\alpha-\lfloor\alpha\rfloor}}{\frac{1}{\alpha-\lfloor\alpha\rfloor}}\right|=\left|\frac{\alpha-\bar{\alpha}}{\bar{\alpha}-\lfloor\bar{\alpha}\rfloor}\right|= \\
=\bar{\alpha} \bar{\alpha}^{\prime}\left|1-\frac{\alpha}{\bar{\alpha}}\right|=\bar{\alpha} \bar{\alpha}^{\prime}\left|\frac{\epsilon}{1+\epsilon}\right|<\bar{\alpha} \bar{\alpha}^{\prime} \frac{\delta}{1-\delta} .
\end{gathered}
$$

An additional way to control the computation is based on the following wellknown and easily verified property of continued fractions.

Lemma 3 If $\beta_{0} / \beta_{1}$ and $\gamma_{0} / \gamma_{1}$ are rational numbers such that

$$
\frac{\beta_{0}}{\beta_{1}}<\alpha<\frac{\gamma_{0}}{\gamma_{1}},
$$


then, as long as the partial quotients of $\beta_{0} / \beta_{1}$ and $\gamma_{0} / \gamma_{1}$ coincide, those partial quotients are the partial quotients of $\alpha$. The first time the partial quotients do not coincide, they provide upper and lower bounds for the correct value.

This result suggests Lehmer's method [7] for reducing the amount of multiprecision work. Assuming that we have a very accurate rational approximation $u / v$ of the real number $\alpha>1$ with very large numbers $u$ and $v$, we can form a suitable lower and upper bound for $u / v$ just by taking the first ten (say) decimal digits of $u$ and $v$ : if $\left\lceil\log _{10} u\right\rceil=k$, take $u_{0}=\left\lfloor u / 10^{k-10}\right\rfloor$ and $v_{0}=\left\lfloor v / 10^{k-10}\right\rfloor$ and choose ${ }^{2}$

$$
\beta_{0}=u_{0}, \quad \beta_{1}=v_{0}+1, \quad \gamma_{0}=u_{0}+1, \text { and } \gamma_{1}=v_{0}
$$

Now we compute partial quotients $a_{0}, a_{1}, \ldots, a_{i_{0}}$ of $\gamma_{0} / \gamma_{1}$ and hence of $\alpha$ as follows:

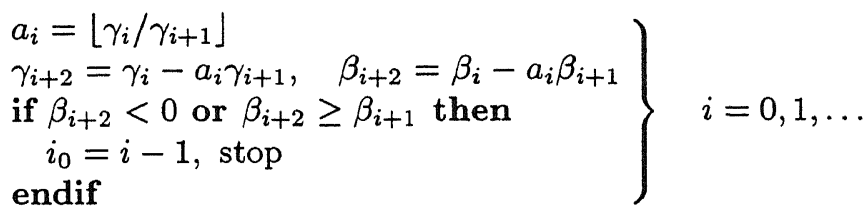

Notice that we do not have to compute the partial quotients of $\beta_{0} / \beta_{1}$ (contrary to what is suggested in [5, p.328]) since as long as $0 \leq \beta_{i+2}<\beta_{i+1}$, we are sure that $a_{i}$ is also the correct partial quotient of $\beta_{0} / \beta_{1}$. After (3) has stopped, we have to update the fraction $u / v$ by acknowledging the computed partial quotients $a_{0}, a_{1}, \ldots, a_{i_{0}}$. If $i_{0}=0$, using $a_{0}$ we replace $u / v$ by $v /\left(u-a_{0} v\right)$. In matrix notation,

$$
\left(\begin{array}{l}
u \\
v
\end{array}\right):=\left(\begin{array}{cc}
0 & 1 \\
1 & -a_{0}
\end{array}\right)\left(\begin{array}{l}
u \\
v
\end{array}\right)
$$

In general, using $a_{0}, \ldots, a_{i_{0}}$ we have

$$
\left(\begin{array}{l}
u \\
v
\end{array}\right):=\left(\begin{array}{cc}
0 & 1 \\
1 & -a_{i_{0}}
\end{array}\right)\left(\begin{array}{cc}
0 & 1 \\
1 & -a_{i_{0}-1}
\end{array}\right) \ldots\left(\begin{array}{cc}
0 & 1 \\
1 & -a_{0}
\end{array}\right)\left(\begin{array}{l}
u \\
v
\end{array}\right) .
$$

The product of the $2 \times 2$ matrices in the right hand side is built up first, and next it is multiplied by the vector $\left(\begin{array}{ll}u & v\end{array}\right)^{T}$, which is the only high-precision computation.

\footnotetext{
${ }^{2}$ If $v_{0}=0$, the first partial quotient of $u / v$ is extremely large, and we have to increase the number of decimal digits in $u_{0}$ and $v_{0}$ accordingly.
} 


\section{The Basic, Polynomial and Direct Methods}

In this section we describe the three methods considered in this study, namely the basic method, the polynomial method, and the direct method derived from Shiu's direct method.

\subsection{The Basic Method}

With the notation of Section 2.1, let $\bar{\alpha}_{i}$ be a rational approximation of $\alpha_{i}$ with relative error bounded by $\delta_{i}$. The basic method for computing the continued fraction expansion of $\alpha=\alpha_{0}$ with safe error control (based on Lemmas 1 and 2) reads as follows.

$$
\begin{aligned}
& a_{i}=\left\lfloor\bar{\alpha}_{i}\right\rfloor \\
& \text { if }\left(a_{i}+1\right) \delta_{i}<\bar{\alpha}_{i}-a_{i}<1-\left(a_{i}+1\right) \delta_{i} \text { then } \\
& \bar{\alpha}_{i+1}=1 /\left(\bar{\alpha}_{i}-a_{i}\right) \\
& \delta_{i+1}=\bar{\alpha}_{i} \bar{\alpha}_{i+1} \delta_{i} /\left(1-\delta_{i}\right) \\
& \text { else } \\
& \text { stop } \\
& \text { endif } \\
& i=0,1, \ldots
\end{aligned}
$$

Since the $\bar{\alpha}_{i}$ are rational numbers, we can use Lemma 3 and (3) to reduce the amount of multi-precision computations. The numbers $\left(a_{i}+1\right) \delta_{i}$ and $\delta_{i+1}$ are computed in (floating-point) single precision. Since (3) works with low-precision approximations $\gamma_{i} / \gamma_{i+1}$ and $\beta_{i} / \beta_{i+1}$ of $\bar{\alpha}_{i}$, some care has to be taken in the check of the inequalities in (4) and in the computation of $\delta_{i+1}$ from $\delta_{i}$ in (4). Here we can use that

$$
\frac{\beta_{2 i}}{\beta_{2 i+1}}<\bar{\alpha}_{2 i}<\frac{\gamma_{2 i}}{\gamma_{2 i+1}}
$$

and

$$
\frac{\gamma_{2 i+1}}{\gamma_{2 i+2}}<\bar{\alpha}_{2 i+1}<\frac{\beta_{2 i+1}}{\beta_{2 i+2}} .
$$

as long as $a_{2 i}$ and $a_{2 i+1}$ are the correct partial quotients of $\bar{\alpha}_{2 i}$ and $\bar{\alpha}_{2 i+1}$, respectively. Detailed information on how this method has been implemented can be obtained from the third author.

From the metric theory of continued fractions it is known [10] that, for almost all $\alpha$, one can compute $p$ partial quotients of $\alpha$ from the first $d$ decimal digits of its decimal representation, where

$$
\lim _{d \rightarrow \infty} \frac{p}{d}=\frac{6 \log 2 \log 10}{\pi^{2}}=0.970 \ldots .
$$

For example, Lochs [9] has computed 968 partial quotients of $\pi$ from its first 1000 decimals, and Brent and McMillan [1] have computed 29200 partial quotients of Euler's constant from 30100 decimals (with $p / d=0.970 \ldots$ ).

A disadvantage of the basic method is that when we have computed as many partial quotients as possible from a given initial approximation of $\alpha$ and then 
wish to compute more partial quotients, we must first compute a more accurate initial approximation, next use the known partial quotients to recompute to the new accuracy the last complete quotient already obtained, and from that extend the list of partial quotients.

\subsection{The Polynomial Method}

Let $\alpha>1$ be an an algebraic number of degree $d>2$ with defining polynomial $f(x)$ (with integral coefficients); that is $f(\alpha)=0$. Say $f(x)$ is reduced if it has the three properties:

(i) its leading coefficient is positive;

(ii) it has a unique simple zero $\alpha>1$;

(iii) its remaining zeros lie in the left half of the unit circle.

The polynomial method [6] for computing the continued fraction expansion of $\alpha$ reads as follows. Set $f_{0}(x)=f(x)$.

$$
\left.\begin{array}{l}
a_{i}=\max \left\{n \in \mathbb{N}, f_{i}(n)<0\right\} \\
g_{i}(x)=f_{i}\left(x+a_{i}\right) \\
f_{i+1}(x)=-x^{d} g_{i}(1 / x)
\end{array}\right\} \quad i=0,1, \ldots
$$

It is easy to see that $f_{1}(x)$ is reduced if $f_{0}(x)$ is and that the unique positive root of $f_{1}(x)$ is given by $1 /\left(\alpha-a_{0}\right)$. It follows that the unique positive root of the polynomial $f_{i}(x)$ is the $i$-th complete quotient of the continued fraction expansion of $\alpha$, and that this algorithm finds the corresponding partial quotients. The time-consuming work lies in the computation of the coefficients of $f_{i+1}(x)$ from those of $f_{i}(x)$ (which grow with $i$ ). The number $a_{i}$ can be computed quickly as follows. If we write $f_{i}(x)=c_{i, d} x^{d}+c_{i, d-1} x^{d-1}+\ldots$, then the sum of the roots of $f_{i}(x)$ is given by $s_{i}=-c_{i, d-1} / c_{i, d}$. Since, for $i \geq 1$, the remaining $d-1$ roots of $f_{i}(x)$ are all located in the left half of the unit circle, the number $s_{i}$ approximates $a_{i}$ with an error not greater than $d-1$; the precise value of $a_{i}$ may be found from $s_{i}$ by trial and error (with an average of $(d-1) / 2$ trials).

It is explained in [2] that (Vincent's Theorem) applying the algorithm to a zero of an arbitrary irreducible polynomial $f(x)$ always rapidly yields a reduced polynomial $f_{i}(x)$.

In [6] it is suggested that the polynomial method may be accelerated as follows. From a low-precision approximation of the real root $>1$ of $f_{n}(x)$, as many as possible ( $m$, say) successive partial quotients are computed with the basic method (and error control). Next, one computes $f_{n+m}(x)$ from $f_{n}(x)$, using $a_{n}, a_{n+1}, \ldots, a_{n+m-1}$, with less computation than is needed to compute the coefficients of all the intermediate polynomials $f_{n+1}(x), \ldots, f_{n+m-1}(x)$. In fact, the coefficients of $f_{i+1}(x)$ are related to those of $f_{i}(x)$ by the transformation (for simplicity, we choose $d=3$ ):

$$
\left(\begin{array}{l}
c_{i+1,3} \\
c_{i+1,2} \\
c_{i+1,1} \\
c_{i+1,0}
\end{array}\right)=-\left(\begin{array}{cccc}
a_{i}^{3} & a_{i}^{2} & a_{i} & 1 \\
3 a_{i}^{2} & 2 a_{i} & 1 & 0 \\
3 a_{i} & 1 & 0 & 0 \\
1 & 0 & 0 & 0
\end{array}\right)\left(\begin{array}{l}
c_{i, 3} \\
c_{i, 2} \\
c_{i, 1} \\
c_{i, 0}
\end{array}\right)
$$


By accumulating the product of the above $4 \times 4$ matrices which we get for $a_{n}$, $a_{n+1}, \ldots, a_{n+m-1}$, and multiplying the resulting matrix with the coefficients of $f_{n}(x)$, we obtain the coefficients of $f_{n+m}(x)$ at the expense of less arithmetic than when we explicitly compute the coefficients of all the intermediate polynomials.

We have carried out some experiments with this acceleration of the polynomial method, but the resulting code is still slower than our implementation of the direct method described in Section 3.3.

Nonetheless, an advantage of the polynomial method is that the computation can always be continued, without any recomputation, provided that we save the exact integral values of the coefficients of the last used polynomial $f_{i}(x)$. To illustrate the growth of these, for $f(x)=f_{0}(x)=x^{3}-8 x-10$, the four coefficients of $f_{100}(x)$ are integers of 68 decimal digits each, and the four coefficients of $f_{1000}(x)$ are integers of $570,571,570$, and 568 decimal digits, respectively.

\subsection{The Direct Method}

The direct method which we formulate here is based on ideas expressed in [2] and [14], combined with error control facilities described in Section 2. The aim is to compute a very good rational approximation of the complete quotient $\alpha_{n+1}$ when the partial quotients $a_{0}, a_{1}, \ldots, a_{n}$ are known, and from that approximation to compute some $n$ partial quotients of $\alpha_{n+1}$. This is done as follows. We have

$$
\alpha=\left[a_{0}, a_{1}, \ldots, a_{n}, \alpha_{n+1}\right]=\frac{\alpha_{n+1} p_{n}+p_{n-1}}{\alpha_{n+1} q_{n}+q_{n-1}},
$$

from which we find, using (1), that

$$
\alpha_{n+1}=\frac{(-1)^{n+1}}{q_{n}\left(p_{n}-\alpha q_{n}\right)}-\frac{q_{n-1}}{q_{n}} .
$$

Now using the mean value theorem and $f(\alpha)=0$, we replace the difference $p_{n} / q_{n}-\alpha$ by $f\left(p_{n} / q_{n}\right) / f^{\prime}\left(p_{n} / q_{n}\right)$, and obtain the approximation

$$
\alpha_{n+1} \approx \frac{(-1)^{n+1}}{q_{n}^{2}} \frac{f^{\prime}\left(p_{n} / q_{n}\right)}{f\left(p_{n} / q_{n}\right)}-\frac{q_{n-1}}{q_{n}} .
$$

The error in this approximation is approximately

$$
\frac{\left|f^{\prime \prime}(\alpha)\right|}{q_{n}^{2}\left|f^{\prime}(\alpha)\right|} \approx \frac{\left|f^{\prime \prime}\left(p_{n} / q_{n}\right)\right|}{q_{n}^{2}\left|f^{\prime}\left(p_{n} / q_{n}\right)\right|} .
$$

From this rational approximation of $\alpha_{n+1}$, partial quotients $a_{n+1}, a_{n+2}, \ldots$, $a_{n+m}, \ldots$ can be computed as long as $q_{n+m}<b q_{n}^{2}$, for some small $b=b(\alpha)>0$. The direct method for computing $N$ partial quotients of the continued fraction expansion of $\alpha$ now reads as follows.

Step 1. Use the basic method (4) to compute a small number of partial quotients and the corresponding partial convergents of $\alpha$, say up to $a_{n}, p_{n}, q_{n}$. 
Step 2. (Check) If $p_{n} q_{n-1}-p_{n-1} q_{n} \neq(-1)^{n+1}$ then stop.

Compute the next rational approximation $\alpha^{\prime}$ of $\alpha_{n+1}$ by

$$
\alpha^{\prime}=\frac{(-1)^{n-1}}{q_{n}^{2}} \frac{f^{\prime}\left(p_{n} / q_{n}\right)}{f\left(p_{n} / q_{n}\right)}-\frac{q_{n-1}}{q_{n}} .
$$

Let $B=b q_{n}^{2}$ for some suitable constant $b=b(\alpha)$.

Compute the next partial quotients $a_{n+1}, a_{n+2}, \ldots, a_{n+m}, \ldots$ with the basic method (4) (using Lemma 3 and (3)) as long as $n+m \leq N$ and $q_{n+m}<B$.

Step 3. Put $n=n+m$; if $n<N$ go back to Step 2 .

The number of partial quotients which can be computed in Step 2 is roughly equal to $n$ so that after the completion of Step 2, the number of partial quotients computed will roughly have doubled compared with before Step 2. Since (7) is very time-consuming, it is worthwhile to choose $n$ in Step 1 such that the last time Step 2 is carried out it starts with a value of $n$ which is slightly larger than $N / 2$. In the beginning of the method the behaviour of Step 2 may be rather erratic; one should therefore compute sufficiently many partial quotients of $\alpha$ in Step 1 to reach the "stable" behaviour phase of Step 2 (an approximate doubling of the number of partial quotients). In practice, this works for $n \approx 100$, but that may depend on the size of the first few partial quotients of the continued fraction of $\alpha$.

\section{Experiments}

We have implemented the three methods described in Section 3 on a SUN workstation, partially in GP/PARI and partially in Magma. The first package is developed by Henri Cohen and his co-workers at Université Bordeaux I, the second comes from John Cannon and his group at the University of Sydney. Initially, we only worked with GP, but at a certain point in the direct method we ran into problems with the stack size, due to the enormous size of the integers involved in this method. Later we learned that these problems can be solved, for example, by programming PARI in Library Mode, but in the meantime we had learned about the Magma package at the University of Sydney and decided to experiment with that. With Magma we did not encounter any stack problems.

In Table 1 we give some timings with Magma and GP for the basic, the polynomial, and the direct methods. Based on these results, we decided to run bigger experiments with our Magma implementation of the direct method.

In Table 2 we give the frequency distributions of the first 200001 partial quotients of the continued fraction of six algebraic numbers, computed by the direct method. For comparison, the last column gives the frequencies of occurrence of partial quotients $j$ :

$$
\log _{2}\left(1+\frac{1}{j}\right)-\log _{2}\left(1+\frac{1}{j+1}\right)
$$


from the well-known Gauss-Kusmin Theorem. Let

$$
K(\alpha, n)=\left(a_{0} a_{1} \ldots a_{n}\right)^{1 /(n+1)}
$$

and

$$
L(\alpha, n)=q_{n}^{1 /(n+1)} .
$$

Then, for almost all $\alpha$,

$$
\lim _{n \rightarrow \infty} K(\alpha, n)=\prod_{k=1}^{\infty}\left(1+\frac{1}{k(k+2)}\right)^{\log k / \log 2}=2.68545 \ldots
$$

and

$$
\lim _{n \rightarrow \infty} L(\alpha, n)=\exp \left(\frac{\pi^{2}}{12 \log 2}\right)=3.27582 \ldots .
$$

The latter fact implies that for almost all $\alpha$ the number of decimal digits in $q_{n}$ is about $n \log _{10} L \approx 0.515 n$. Table 2 gives the values of $K(\alpha, 200000)$ for the six algebraic numbers which we consider. Table 2 also lists the largest partial quotient $a_{n}$ found, and the corresponding index $n$. Only in case (A) is there an early occurrence of a large partial quotient $\left(a_{121}=16467250\right)$, but soon after that the expansion settles down and no further extremely large partial quotients occur. To illustrate this, Table 3 lists $a_{n}$ for $n=0, \ldots, 200$ and for $n=199901, \ldots, 200000$. The "abnormal" initial behaviour is explained in [15].

Table 4 presents, for some values of $n$, the number of decimal digits in $q_{n}$ and that number divided by $n$. The values of $n$ in Table 4 are those for which the direct method computes a new rational approximation of $\alpha_{n}$ : it illustrates the approximate doubling of these $n$-values, especially for larger values of $n$. The last column shows good convergence to the expected value $C_{1}=\pi^{2} /(12 \log 2 \log 10)=0.51532 \ldots$.

\section{Conclusion}

We have compared three different methods (the basic, the polynomial, and the direct method) for computing the continued fraction expansion of algebraic numbers, and observed that the direct method is the most efficient one in terms of CPU-time and memory, at least for our implementations (in GP/PARI and Magma). We have applied the direct method to the computation of 200,001 partial quotients of six different algebraic numbers, and found no apparent deviation from the theory of Khintchine and Lévy, which holds for almost all real numbers. 


\section{Acknowledgment}

Part of this research was carried out while the third author was visiting the ceNTRe for Number Theory Research at Macquarie University, Sydney, and the Computer Sciences Laboratory of the Australian National University, Canberra, in September-November 1995. He thanks the first and the second authors for their hospitality. We also wish to acknowledge the help of Wieb Bosma of the University of Sydney with the use of the Magma package.

\section{References}

1. Richard P. Brent and Edwin M. McMillan, 'Some new algorithms for high-precision computation of Euler's constant', Math. Comp. 34 (1980), 305-312.

2. Enrico Bombieri and Alfred J. van der Poorten, 'Continued fractions of algebraic numbers', in Computational Algebra and Number Theory, Sydney 1992, Wieb Bosma and Alf van der Poorten eds., (Kluwer, 1995), 137-152.

3. David G. Cantor, Paul G. Galyean and Horst G. Zimmer, 'A continued fraction algorithm for real algebraic numbers', Math. Comp. 26 (1972), 785-791.

4. A. Khintchine, 'Metrische Kettenbruchprobleme', Compositio Math. 1 (1935), 361382.

5. Donald E. Knuth, The Art of Computer Programming, Volume 2, Seminumerical Algorithms, (Reading, Mass.: Addison-Wesley, Second Edition, 1981).

6. Serge Lang and Hale Trotter, 'Continued fractions for some algebraic numbers', $J$. reine angew. Math., 255 (1972), 112-134.

7. D. H. Lehmer, 'Euclid's algorithm for large numbers', Amer. Math. Monthly, 45 (1983), 227-233.

8. P. Lévy, 'Sur le développement en fraction continue d'un nombre choisi au hasard', Compositio Math. 3 (1936), 286-303.

9. Gustav Lochs, 'Die ersten 968 Kettenbruchnenner von $\pi$ ', Monatsh. Math., 67 (1963), 311-316.

10. Gustav Lochs, 'Vergleich der Genauichkeit von Dezimalbruch und Kettenbruch', Abh. Math. Seminar Hamburg, 27 (1964), 142-144.

11. Attila Pethö, 'On the resolution of Thue inequalities', J. Symb. Comp. 4 (1987), 103-109.

12. R. D. Richtmyer, Marjorie Devaney and N. Metropolis, 'Continued fraction expansions of algebraic numbers', Numer. Math. 4 (1962), 68-84.

13. A. Schönhage, 'Schnelle Berechnung von Kettenbruchentwicklungen', Acta Informatica 1 (1971), 139-144

14. P. Shiu, 'Computation of continued fractions without input values', Math. Comp. 64 (1995), 1307-1317.

15. H. M. Stark, 'An explanation of some exotic continued fractions found by Brillhart', in Computers in Number Theory, A. O. L. Atkin and B. J. Birch eds., (Academic Press, 1971), 21-35.

16. Benjamin M.M. de Weger, Complete solution of a Thue inequality, Technical Report 9561/B, December 15, 1995, Econometric Institute, Erasmus University Rotterdam. 